\title{
Gene Delivery Systems: Recent Progress in Viral and Non-Viral Therapy
}

\author{
Erdal Cevher, Ali Demir Sezer and Emre Şefik Çağlar
}

Additional information is available at the end of the chapter

http://dx.doi.org/10.5772/53392

\section{Introduction}

Thanks to the changes in medicine, pharmacological treatment rapidly progresses into new fields. There is an emphasis on the development of treatment methods to eliminate underlying factors rather than to treat the symptoms of a disease. Therefore, research is increasingly utilizing knowledge from the field of genetics. Genetic mutation and deletion lead to many genetic disorders. Genetic disorders in metabolic pathways, regulation of cell cycle, ligand/receptor function, cell skeleton and extracellular proteins cause serious diseases (Yaron et al., 1997).

Both genetic and acquired diseases may be treated using gene therapy. Genetic diseases are generally caused by deletion or mutation of a single cell. Conversely, in acquired diseases, a single gene cannot be defined as the sole cause of a disease. Gene therapy has increased in prominence, and has shown great potential in treating acquired and genetic diseases (Conwell and Huang, 2005).

Gene delivery systems are categorized as: viral-based, non-viral-based and combined hybrid systems. Viral-mediated gene delivery systems consist of viruses that are modified to be replication-deficient, but which can deliver DNA for expression. Adenoviruses, retroviruses, and lentiviruses are used as viral gene-delivery vectors (Escors and Breckpot, 2010).

Non-viral gene delivery systems were introduced as an alternative to viral-based systems. One of the most important advantages of these systems is improved transfection. Non-viral systems are categorized according to preparation, as physical or chemical types. The most common physical methods are microinjection, electroporation, ultrasound, gene gun, and hydrodynamic applications. In general terms, physical methods refer to delivery of the gene via the application of physical force to increase permeability of the cell membrane. In contrast, chemical methods utilize natural or synthetic carriers to deliver genes into cells. In 
this method, polymers, liposomes, dendrimers, and cationic lipid systems are used as gene delivery systems (Miyazaki et al., 2006; Prokop and Davidson, 2007).

\section{Gene therapy}

This chapter provides general information on gene delivery systems, how they are used, their relative merits, and selection of the most appropriate methods for new studies on gene therapy.

The drug sector is entering a new era that will enable treatment of the underlying cause rather than the symptoms of a disease. Many human diseases result from mutations or deletions, in genes, which lead to disorders in metabolic pathways, ligand/receptor function, cell cycle regulation, cell skeleton or extra-cellular protein structure and function (Sullivan, 2003). With gene therapy, the disease can be treated by the injection of exogenous nucleic acid sequences designed to target the diseased tissues of the body (Yaron et al., 1997).

Diseases that can be treated by gene therapy are categorized as either genetic or acquired. Genetic diseases are those which are typically caused by the mutation or deletion of a single cell. Conversely, a single gene is not defined as the sole cause of acquired diseases. Although gene therapy was initially used to treat genetic disorders only, it is now used to treat a wide range of diseases such as cancer, peripheral vascular diseases, arthritis, neurodegenerative disorders and AIDS (Mhashilkar et al., 2001). The expression of a single cell, directly delivered to the cells by a gene delivery system can potentially eliminate a disease. Prior to gene therapy studies, there was no alternative treatment for genetic disorders. Today, it is possible to correct genetic mutation with gene therapy studies (Sullivan, 2003).

\subsection{Chronology}

The term gene therapy was first introduced at the International Congress of Genetics (Yaron et al., 1997). The techniques utilized by Gregor Mendel in the 1850s, which were then developed by Ronald Fischer at the beginning of the twentieth century, formed the turning points in genetics. The work of both Mendel and Fischer laid the foundations of genealogy. The material they studied was later termed as gene by the Danish botanist Wilhelm Johannsen (Yaron et al., 1997). Towards the end of the 1970s, the background of the majority of genetic disorders was understood, and gene therapy came to the fore. The first gene therapy trial in humans was conducted at the beginning of the 1970s, and it was observed that naturally occurring DNA and RNA tumor viruses successfully delivered new genetic information to the genomes of mammal cells (Escors and Breckpot, 2010).

Due to developments in the science of genetics, at the beginning of the twentieth century, it was understood that diseases such as hemophilia were genetic diseases. Similarly, it was found that diseases such as colon cancer, diabetes, and retinoblastoma were also geneticbased diseases. In the 1980s, gene transfer to mammalian cells came to the fore after the 
development of retroviral vectors (Yaron et al., 1997). In the mid-1980s, gene transfer to mammalian cells became a routine procedure. Retrovirus-based gene therapies brought significant advantages, as they can stably integrate their genomes to host-cell chromosomes. After the 1980s, DNA was defined as a genetic material. Later, it was structurally analyzed and further advances allowed the modification of genetic code. These discoveries on genetic material made cloning possible (Escors and Breckpot, 2010).

In 2006, melanoma was successfully treated for the first time. The treatment used a retroviral formulation of melanoma antigen (MART-1)-specific T-cell receptor (TCR), which encodes $\alpha$ - and $\beta$ - chains. In recent years, great advances have been made in gene therapy, ranging from somatic cloning and completion of human genome project to the discovery of microRNA-based gene-regulating systems (Escors and Breckpot, 2010).

The use of non-viral gene delivery systems in gene therapy also significantly increased in recent years. Naked plasmid DNA, coated with gold particles was effectively introduced to cells using the non-viral gene gun technique. This technique was first used in plant cells in 1987, and is today commonly used in mammal cells and tissues. One of the most successful current gene therapy techniques is hydrodynamic injection (Willemejane and Mir, 2009).

\subsection{Gene delivery systems}

Gene delivery systems use various methods to allow uptake of the gene that has been selected to target the cell (Conwell and Huang, 2005). The successful design of a gene delivery system requires complete understanding of the interaction mechanism between the target cell and delivery system. Understanding intercellular traffic and targeting mechanism is the most important factor in designing a more effective gene delivery system. Cell targeting refers to delivery of the therapeutic agent to a specific compartment or organelle of the cell. It is the most commonly used mechanism in endocytosis gene therapy, particularly in cellular uptake of non-viral gene delivery systems (Prokop and Davidson, 2007). After the cellular uptake of the delivery system by endocytosis, cellular release takes place to initiate DNA transcription and translation, and to produce the related protein. A successful gene delivery procedure involves minimizing potential inhibitory inflammatory response while also overcoming certain barriers at each step of the gene delivery procedure, in order to optimize gene activity (Conwell and Huang, 2005).

Viral gene delivery systems consist of viruses that are modified to be replication-deficient which were made unable to replicate by redesigning which can deliver the genes to the cells to provide expression. Adenoviruses, retroviruses, and lentiviruses are used for viral gene delivery. Viral systems have advantages such as constant expression and expression of therapeutic genes (Sullivan, 2003). However, there are some limitations that restrict the use of these systems, which particularly include the use of viruses in production, immunogenicity, toxicity and lack of optimization in large-scale production (Witlox et al., 2007) (Figure 1). 


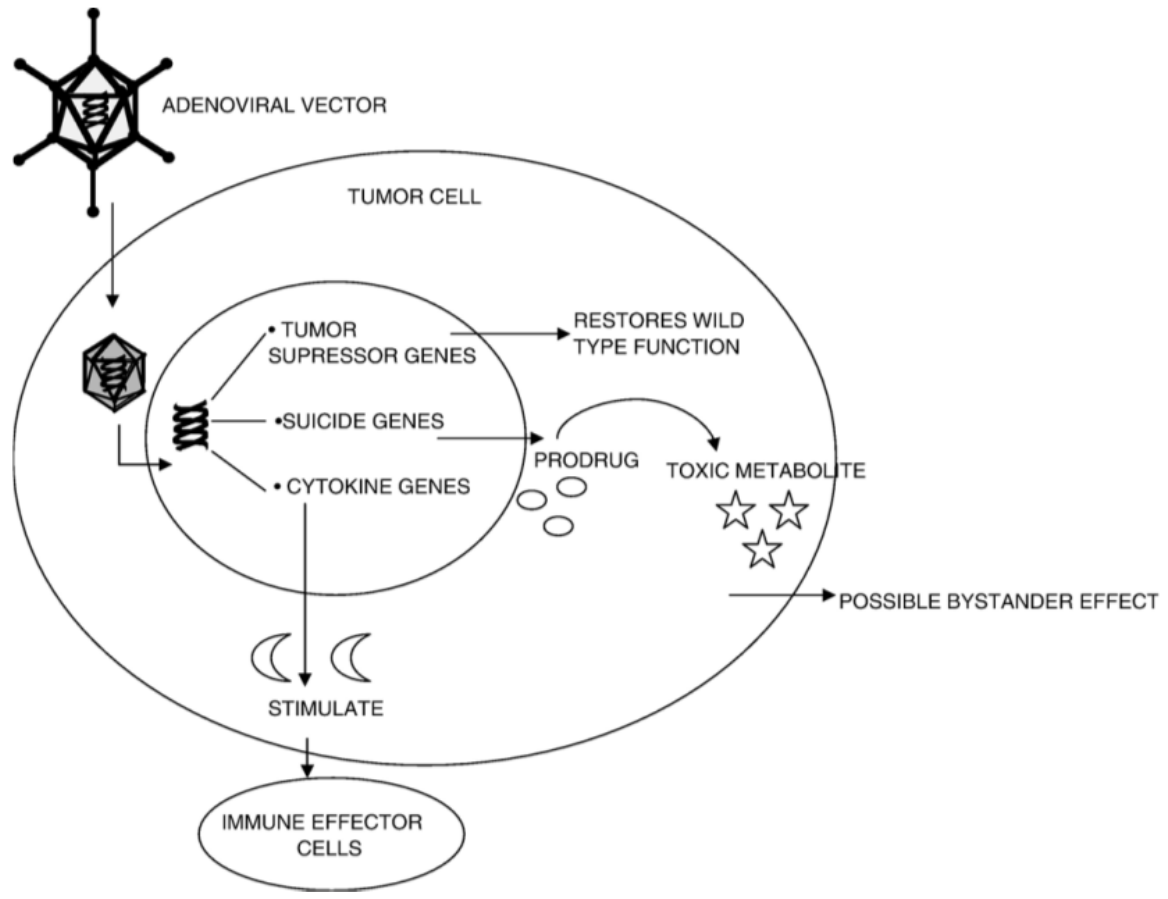

Figure 1. Gene therapy strategies; mutation compensation, suicide gene therapy and immunopotentiation (Witlox et al., 2007).

Non-viral gene delivery systems were developed as an alternative to viral-based systems. One of the most important advantages of these systems is that they develop transfection. Non-viral gene delivery systems are divided into two categories: physical and chemical. Microinjection, electroporation, gene gun, ultrasound-mediated methods, and hydrodynamic systems are the most widely used physical methods. Physical methods involve the use of physical force to increase the permeability of the cell membrane and allow the gene to enter the cell. The primary advantage of physical methods is that they are easy to use and reliable. However, they also have the disadvantage of causing tissue damage in some applications.

Chemical methods involve the use of carriers prepared from synthetic or natural compounds for gene delivery into the cell, including synthetic and natural polymers, liposomes, dendrimers, synthetic proteins, and cationic lipids. The biggest advantages of these systems are that they are non-immunogenic and generally have low toxicity.

\subsubsection{Viral gene delivery systems}

Since a large number of viruses have appropriate mechanisms for transfer of genetic material to the target cell, current gene technologies concentrated on the use of viral vectors that provide high transduction effectiveness and advanced level of gene expression. The 
optimal design of a viral vector depends on the types of virus to be used (Wunderbaldinger et al., 2000).

For safe application of in vivo gene therapy, firstly some problems should be eliminated. Therapeutic factors should only be expressed in related cell types, and should not cause any undesired effect in healthy cells (Yaron et al., 1997).

\subsubsection{Adenoviral systems}

Adenoviruses (Ad) were first discovered in 1953 by isolation from human adenoid tissue cultures (Campos and Barry, 2007; Majhen and Ambriovic-Ristov, 2006). They are commonly used as gene vectors (Dinh et al., 2005). C group adenoviruses Ad2 and Ad5 are the most widely studied adenoviruses. The capsid of an adenovirus determines virus tropism. Groups A and C-F first bind to highly-expressed coxsackie virus B-adenovirus receptor, and thus realize their high infectivity in many tissues. In contrast, group B binds to complement-regulatory protein CD46. Adenoviruses are replicated and produce virions, which contain the nucleus of the infected cell (Dinh et al., 2005). These vectors have the ability to be replicated, and purification of the vectors generally involves easier and shorter processes (Armendariz-Borunda et al., 2011).

Adenoviruses are well-characterized, non-integrated, $\sim 26-40 \mathrm{~kb}$ in length, relatively large, non-enveloped, linear dsDNA viruses coated with icosahedral particle, with a diameter of $950 \AA$ (excluding elongated fiber proteins) and a molecular weight of approximately 150MDa. They are unreplicated, and infect cells quickly. Adenoviral particles do not contain lipid or membrane; therefore they are stable in solvents such as ether or ethanol. The use of adenoviruses, which are one of the first developed vector systems for gene expression, is a great discovery (Campos and Barry, 2007).

Adenoviruses have important characteristics, which make them indispensable for gene transfer. The most important ones are their well-known molecular biology; delivery capacity of foreign DNA fragments up to $36 \mathrm{~kb}$; and ability to transfect DNA into many cell types (Sullivan, 2003).

Adenoviruses are one of the largest and most complex viruses, whose Ad structure was analyzed with cryo-electron microscopy and X-ray diffractometry. An Ad genome of approximately $36 \mathrm{~kb}$ codes more than forty genomes; however, only 12 of these were demonstrated as the component of the virus particle. As seen in Figure 2 it was reported that crystal structures of single Ad proteins contained fiber knob, shaft, domains, penton base, hexon, and cysteine protease. Ad capsid consists of 252 sub-units called capsomeres, which contain 240 hexonproteins and 12 of the penton base. In addition, the capsid contains pIIIa, pVI, PVIII, and pIX proteins. Each of the 12 capsid corners contains penton bases wrapped by 5 hexons. The penton base serves as a hook for the fiber protein, which projects from the virus like an antenna. Fiber is a homotrimer, where 3 identical polypeptides bind in the same direction, and which contains 3 structurally and functionally different domains: Nterminus, which binds the fiber non-covalently to the penton base; C-terminus, which gives the disc-like knob form, which is responsible for binding to the receptor; and the rod-like 
shaft, which varies in length according to the serotype of the virus (Medina-Kauwe LK. 2003; Majhen and Ambriovic-Ristov, 2006).

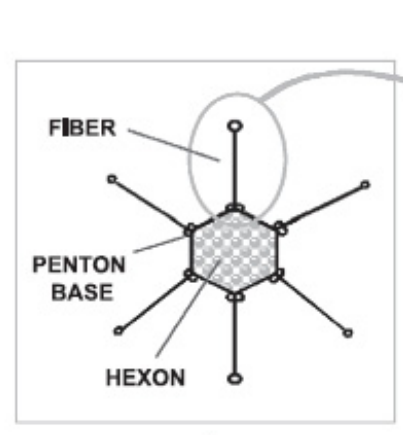

A

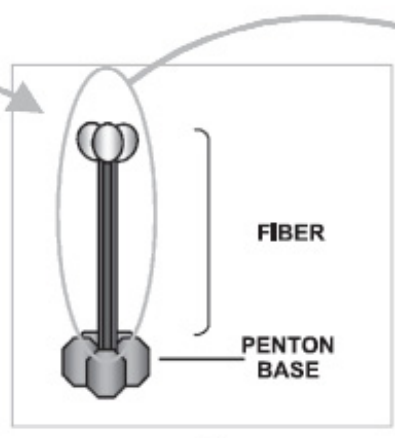

B

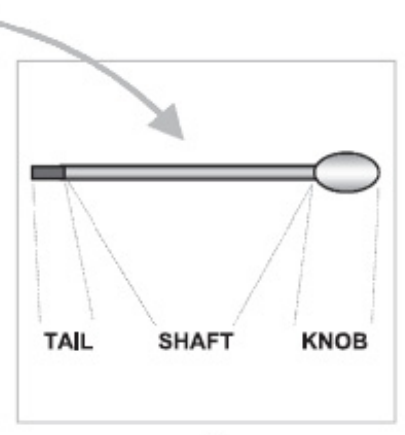

C

Figure 2. Schematic of the adenovirus capsid. (A) Whole capsid identifying fiber, penton base, and hexon. (B) Enlargement of circled region in (A), showing homotrimeric fiber bound to homopentameric penton base. (C) Fiber monomer, identifying tail, shaft, and knob domains. Pictures are not drawn to scale (Medina-Kauwe LK. 2003).

A viral genome is in a condensed state inside cell nucleus, together with protein V, VII and $X$, and the $5^{\prime}$ terminus of the AdDNA is covalently linked to a terminal protein (TP). The terminal protein (TP) functions as the initiation primer in viral DNA replication. An $\mathrm{Ad}$ genome consists of 5 early (E1A, E1B, E2, E3 and E4), 4 interim (IVa2, IX, VAI, VAII) and one late transcriptional unit. E1A is the first viral transcriptional unit, which will be expressed after entering the cell. E1A proteins activate other transearly transcription units, which will create an optimum medium for virus replication by causing the products to enter in $S$ phase of the cell E1B proteins bind to p53, Bak, and Bax proteins, and allows the infected cell survive by inhibiting p53 dependent apoptosis. The E2A unit encodes the proteins that function in viral genome replication, including DNA polymerase, prethermal protein, and single-stranded DNA-binding protein. While the E4 transcription unit encodes the proteins that affect cell cycle control and transformation, E3 protein disrupts the immune response of the host and ensures the continuation of the infected cell (Majhen and Ambriovic-Ristov, 2006).

The nucleus is surrounded by an icosahedral capsid with a symmetrical structure, formed by the non-covalent interactions between 7 proteins (II, III, IIIa, IV, VI, VIII, and IX) (Smith et al., 2010). Adenoviral structural proteins are responsible for the stabilization of genome and encapsidation of the nucleoprotein nucleus. The icosahedral capsid consists of seven peptides:trimerichexons, which are found in complex form with three minor capsid polyproteins (VI, VIII and IX), which provides stabilization (II); pentonbase (III); receptor binding fiber (IV) and penton-bound protein which serves as a bridge between protein and the hexon base (III). The fiber consists of 3 domains which are tail in N-terminus, rod-like shaft and globular knob in C-terminus (Couglan et al., 2010). Adenoviruses enter target cells via receptor-mediated endocytosis. Instead of combining its own DNA with the genomes of 
the host cell, the adenovirus, remains as an episome within the infected cell. This provides high efficiency (10); however, it should not be ignored, since it will restrict the longevity of expressed trans genes; in other words, protein expression will quickly cease (Yaron et al., 1997; Muzzonigro et al., 1999).

Penton and fiber proteins of virus capsid interact with the coxsackievirus-adenovirus receptor cell surface protein to provide cell binding. These proteins are then localized in clathrin-coated pits through NPXY motive of $\beta_{3}$ and $\beta_{5}$ integrin sub units of the cell. Dinamine, which is systolic GTPase, mediates cellular uptake of the virus into the cell via endocytosis by providing construction and development of clathrin-coated pits. Viral capsid proteins dissociate prior to endocytosis, and the $\mathrm{pH}$ value of the viral endosome decreases due to proton pumps. At $\mathrm{pH}$ 6.0, the virus has the ability to detach from the vesicle and enter the cytosol. Capsid proteins of the virus move towards the nucleus through microtubules and fragment in the following stages. Although a considerable part of the capsid proteins remain in the periphery of the nucleus, viral DNA crosses via nuclear pores (Ziello et al., 2010).

For successful delivery of DNA to the nucleus, viruses must facilitate cell-specific binding, endocytosis internalization, propagation from endocytic vesicles to cytosol, delivery into cytoplasm, translocation from one terminus of the nuclear envelope to other, and finally expression of the delivered gene (Dinh et al., 2005).

Direct injection or bolus delivery through inhalation is the easiest form of viral delivery. However, since the virus will spread from the injection area, a high dose is required to achieve therapeutic effectiveness. The spread of the virus from the injection site limits the regional effectiveness and immune response. Adenoviral vectors can be targeted via selective modification of coat proteins. There is a relatively high level of protein expression following the transduction, equal to approximately 35\% of total cellular protein (Zhang et al., 2009).

Adenoviruses have many natural properties that enable them to be used as a vector for on colitic, vaccine, or gene therapy. Non-enveloped viruses can be kept in lyophilized form in a stable state inside a flacon tube or capsule; they can be transported without cold chain; by mediating in high transduction effectiveness in dividing and non-dividing cells, they can form 104 virus particles per infected cell (Khare et al., 2011). Adenoviruses achieve suitable transduction through a high level of expression and become beneficial in in vivo conditions (Muzzonigro et al., 1999).

Adenoviruses have been one of the most promising methods for high effectiveness in in vivo gene therapy. For example, following systemic vector delivery, transduction levels of hepatocytes were observed by adenoviral systems. These are some of the most effective vectors for gene delivery to various organs; however, they have very significant disadvantages. Some target cells have low ratios of appropriate primary and/or secondary adenoviral receptors, and this requires a high dose of vector application to cause target-cell cytotoxicity. Additionally, non-discriminating tropism of the virus can also lead to transduction to untargeted cells (Reynolds et al., 1999). The most serious problem in the use of Ad vectors is their tendency to cause strong immune and inflammatory responses at high doses (Navarro et al., 2008). 


\subsubsection{Retroviral systems}

Retroviruses are diploid, single-stranded, circular-enveloped RNA viruses of the family Retroviridae, with a genome of 7-11 kb, and a diameter of approximately 80-120 nm (Osten et al., 2007; Escors and Brecpot, 2010). The Retroviridae family is divided into two subfamilies: Orthoretrovirinae and Spumaretrovirinae species (Osten et al., 2007). Retroviruses cause diseases such as AIDS, leukemia, and cancer; however, their use as a vector in gene therapy brought new developments in treatment (Pages and Danos, 2003).

The ability of retrovirus-based gene delivery vectors to carry foreign genetic material was first realized in the early 1980s (El-Aneed, 2004; Escors and Brecpot, 2010). Retroviruses are viruses that integrate with host genome to produce viral proteins (gag, pol, env) that are extracted during gene delivery (El-Aneed, 2004). Retroviral vectors have the capacity to delivery DNA up to $8 \mathrm{~kb}$ (Navarro et al., 2008). The most commonly used retroviruses are the Moloney murine leukemia virus species, which have the capacity to deliver exogenous genetic material up to approximately $9 \mathrm{~kb}$ (Muzzonigro et al., 1999).

A virion nucleus consists of circular or triangular gag-encoded capsid proteins. They are coated with gag-encoded nucleocapsid protein. The nucleus contains pol-encoded enzymes, reverse transcriptase, and integrase. Retrovirus genomes have various characteristics. For example, these viruses are diploid in the real sense, and their genomes are produced by cellular transcriptional mechanism. In addition, these viruses require cellular (tRNA) for replication (Luban, 2000). The genome of these (+) sense RNA viruses is not directly processed as mRNA immediately after infection Considering the structure of the genome, retroviruses are categorized as either simple or complex forms. Simple and complex retroviruses encode gag (group-specific antigen), pro (protease), and pol (polymerase) genes; complex retroviruses also encode a large number of additional genes. Retroviruses were the first viruses to be modified for gene delivery, and have also been used in the majority of clinical trials of gene therapy (Hu and Pathak, 2000).

Most of retroviral vectors used in clinical studies are based on the Moloney murine leukemia virus (MMLV). MMLV is a well-studied and characterized virus; its genome encodes three polyproteins that are required in trans form for replication and packaging: gag, pol and env (Escors and Brecpot, 2010).

An ideal retroviral vector for gene delivery should be cell-specific, regulated, and safe. Effectiveness of delivery is important, as it will also determine the effectiveness of therapy (Hu and Pathak, 2000). Retroviruses have a lipid envelope. In order to enter a host cell, they use the interactions between cellular receptors and virally encoded proteins, which are embedded in the membrane (Hu and Pathak, 2000). Combination of chimeric envelope protein with viral particles allows the retrovirus to bind receptor-positive target cells (Yi et al., 2011). SU in the virion surface binds to CD, which is a cell-surface protein expressed by some T-cells and is found in macrophages. Binding to CD4 induces some changes in the envelope protein (SU) of the domain of epidermal growth factor (EGF), which allows for interaction with chemokine receptors (CKR). CKRs are a family of cell-surface-G-proteins functioning as receptors to stain molecules called chemokines. 
Infusion of viral and cellular membranes starts the internalization of the viral nucleus (Yi et al., 2011); the viral envelope combines with the cell membrane. Envelope glycoprotein (Env) of retroviruses is responsible for determining tropism. Binding of Env to cellular receptor and fusion of viral and cellular membranes are the first stages of infection. Trials that targeted retroviruses by adding single-stranded antibodies or Env cell-binding ligands showed limited success. The addition of such large ligands to Env protein prevents Env from binding to the virion (Bupp and Roth, 2002). Env protein takes a particle-coated structure and releases the viral nucleus into the cytoplasm. Single-stranded DNA gets reversely transcripted to double-stranded DNA in the nucleus (Osten et al., 2007; Karlsson et al., 1987). Viral RNA is reverse transcribed to a DNA copy using virally-encoded reversetranscriptase enzyme in the virion (Hu and Pathak, 2000). Retroviruses follow a different replication cycle through transformation of single-stranded RNAs into double-stranded DNA in the infected cell. Viral DNA synthesis takes place in cytoplasm by virion-dependent DNA polymerase, which is termed reverse transcriptase. Viral DNA enters the nucleus, where takes the ring form for the first time, and serves as a draft for RNA synthesis (Olsen and Swanstorm, 1985). The first DNA product is a linear double layer molecule, which forms during DNA synthesis. Within the nucleus, some linear DNA is turned into many different forms of ring DNA; these mainly contain two copies of LTR units in tandem, or a single copy of LTR unit (Olsen and Swanstorm, 1985). The end of each LTR unit creates short, deficient repetitions; this represents the strand domain required for this integration (Olsen and Swanstorm, 1985), and is delivered to the nucleus. This delivery takes place through cell division for oncoretroviruses, and through active transport for lentiviruses. Here, lentiviruses are observed to be more advantageous than oncoretroviruses, as they have the ability to deliver genes to non-dividing cells. When viral DNA enters the nucleus, it integrates with the DNA of the host cell (provirus). Retroviruses introduce their genetic material to the host cell genome in a stabile manner during mitotic division. Since these types of retroviruses only transfer genes to dividing cells, many procedures that utilize retroviruses are applied ex vivo. However, more recent lentiviral-based retroviral vectors reduce these restrictions (Muzzonigro et al., 1999). Most retroviruses infect cells that can be actively divided during mitotic division. This property protects normal tissue, and although it naturally targets the tumor, all tumors contain non-dividing cells in G0, which is the resting phase of the tumor cell cycle (Hu and Pathak, 2000; Kitamura et al., 2003; El-Aneed, 2004) (Figure 3).

Infected cells are then transcribed and spliced. Full-length viral RNA (which is the RNA that encodes all proteins) is delivered to cytoplasm and translated; other cellular procedures are modification and delivery of RNA from the nucleus (Hu and Pathak, 2000). Spliced fulllength viral RNA is packaged into viral particles. Retroviruses are RNA viruses that are replicated over integrated DNA intermediate products. Retroviral particles surround two copies of full-length viral RNA, containing all of the genetic information required for virus replication, with capsid (Hu and Pathak, 2000). Virologic and genetic studies indicated that specific packaging of retroviral genomic RNA was carried out through the interaction with the nucleocapsid (NC) domain of Gag polyprotein. Retroviral NC domains are generally very simple, and contain one or two zinc knob motifs consisting of $\mathrm{C}-\mathrm{C}-\mathrm{H}-\mathrm{C}$ sequences. Zinc knobs form metal-coordinated reverse turn, stabilized by NH-S hydrogen bonds. Most retroviral zinc knobs contain a hydrophobic cut on the surface of the mini-globular domain, 


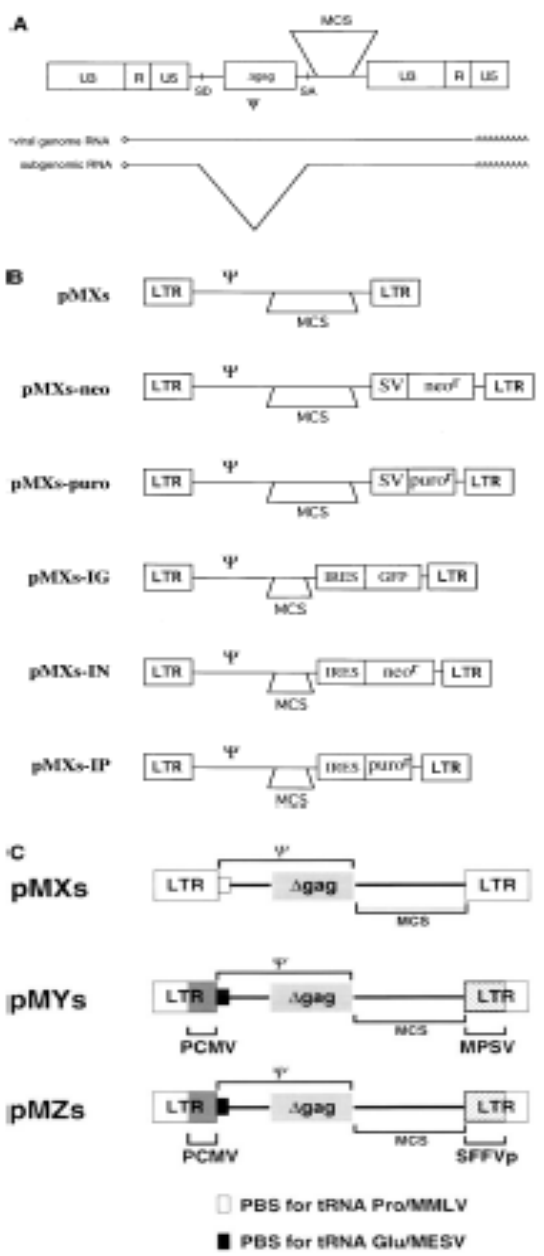

Figure 3. Structures of retrovirus vectors. (A) Basic structure of retrovirus vectors and two transcripts from the vector. In the replication competent MMLV, the Gag-Pol and the Env proteins are translated from the genomic RNA and the subgenomic RNA, respectively. The subgenomic RNA is a spliced form RNA, and the splicing occurs from the splice donor site (SD) to the splice acceptor site (SA). Both $5^{-}$ and $3^{-}$LTRs consist of U3, R, and U5 regions. $\Psi$ = packaging signal; $\Delta$ gag = truncated gag sequence. (B) Structures of versatile pMXs-derived vectors. $\mathrm{LTR}=$ long terminal repeat; $\Psi$ = packaging signal; MCS = multi-cloning site; IRES = internal ribosomal entry site; GFP = green fluorescent protein' neor $=$ neomycin-resistant gene; puror $=$ puromycin-resistant gene. (C) Structures of pMXs, pMYs, and pMZs vectors. The $5^{-}$LTR, primer binding site (PBS), and the extended packaging signal of pMXs are derived from the MFG vector. PBS used in PMYs and pMZs are derived from MESV (murine embryonic stem cell virus) and binds tRNA-glu instead of tRNA-pro. MCS is designed for cDNA library construction and is preceded by triple stop codons (not shown). White box = MMLV LTR; gray box = PCMV LTR; hatched box = MPSV LTR or SFFVp LTR. MCS = multi-cloning site (Kitamura et al., 2003). 
recognizing the specific structure of RNA or DNA. Base $\mathrm{N}$ - and C- terminal tails of nucleocapsid domains are conformationally indurable (Miyazaki et al., 2011). Retroviral genome packaging is generally localized between the binding donor (SD) region and the gag start codon in the $5^{\prime}$ head region. It should be noted that the packaging sign generally coincides with the dimerization region, which indicates that packaging combines with genome dimerization (Miyazaki et al., 2011). Inhibition of genome dimerization through addition or deletion of mutations at dimer initiation sites (DIS) causes a significant decrease in genome packaging. Studies of mutant viruses containing two retroviral genomes showed that these were non-covalently dimerized in progen viruses. $5^{\prime}$ untranslated region (UTR) packages monomeric genome, which indicates that the genome packaging procedure occurs via the interaction of two 5' UTR (Miyazaki et al., 2011). Virion maturation occurs by the budding of the particle from the cell (Escors and Brecpot, 2010). The translational mechanism of the host synthesizes and modifies viral proteins. Newly synthesized viral proteins and full-length RNAs combine to produce a new virus form, which will be budding from the host cell (Hu and Pathak, 2000).

A retrovirus infects the target cell by providing interaction between viral envelope protein and cell surface receptor on the target cell. The virus then internalizes to the place where its single-stranded RNA turns into double-stranded DNA. Double-stranded DNA is delivered to the nucleus and integrated to the host cell genome there. Many types of retrovirus types require degradation of mitosis and then the nuclear envelope for the arrival of a viral genome within the nucleus (Robbins and Ghivizzani, 1998).

Stable binding of viral DNA to the host genome is advantageous because it will provide long-term expression of transgenes required for therapeutic effect. However, one of the disadvantages of current retroviral transfer systems is that they are not specific to types of target cells (Yi et al., 2011).

One of the most important properties of retroviruses is that they can integrate a reverse transcribed genome to the host cell chromosome (Miyazaki et al., 2011).

Unlike adenoviral vectors, retroviral vectors realize transfection through transgene integration to the target cell genome. However, transgene expression ceases within a few days or weeks. Retroviral vectors reveal gene transfer that is unsuitable for many cells in in vivo conditions, and this partly stems from rapid inactivation of the human complement system. This silencing is not well characterized; however, it appears as a result of methylation in DNA or promoter regions, or participation of splice region to condensed chromatin (Reynolds et al., 1999; Navarro et al., 2008).

The ability of retroviruses to enter their genomes to host DNA enables them to make stable modifications to the life cycle of the host cell. This stability is greater than viruses such as adenovirus, herpes simplex virus (HSV), and papilloma virus, which remain episomal (Robbins and Ghivizzani, 1998).

\subsubsection{Lentiviral systems}

In recent years, studies mainly concentrated on the use lentiviral vectors. Lentivirals are viral systems without small, retrovirus-like viral proteins and no capacity for replication; 
they provide gene delivery to non-dividing cells. This characteristic property is an advantage for various gene therapy applications used in targeting in post-mitotic and highly differentiated cells. The most important advantage of lentiviruses compared with other retroviruses is their ability for gene transfer to non-dividing cells (Escors and Brecpot, 2010; Yi et al., 2011; Matrai et al., 2009; Freed, 1997). This characteristic is believed to be related to the pathogenic characteristics of lentiviruses, which infect terminally differentiated cells of monocyte/macrophage lineage (Freed, 1997). For this reason, lentiviral vectors can be used for transgene expression to neuron cells (Yi et al., 2011).

Genome of lentiviruses have a more complicated structure; they contain accessory genes which regulate viral gene expression, control combination of infectious particles, modulate viral replication in infected cells, and are associated with the continuance of infection (Howarth et al., 2010). In other words, the fact that it contains regulatory and accessory genes apart from gag, pol and env, requires lentiviruses to adopt a more complex form (Osten et al., 2007). For example, HIV-1 is one of the most widely used lentiviral vectors, and contains six accessory genes (tat, rev, vif, vpr, nef, vpu) (Osten et al., 2007; Yi et al., 2011). These proteins are involved in all steps of cell cycles, which are termed: budding, maturation, and integration. In addition to helping combination of virions, these proteins ensure nuclear export and transcription ratio of RNA (Yi et al., 2011).

HIV infects CD4-positive T lymphocytes and macrophages (CD4 antigen acts as a primer surface receptor for HIV-1), and causes chronic immune deficiency, known as acquired immune disorder syndrome (AIDS). HIV-1 genome additionally contains 2 regulatory genes (tat and rev), and 4 accessory genes (vpr, vpu, vif, nef). The lenti viral genome is $9.2 \mathrm{~kb}$ in length. Tat protein increases transcriptional activity in 5' LTR of integrated provirus from promoter regions. Rev protein transports non-spliced HUV mRNA from the nucleus. In general, accessory proteins increase virulence against the host organism. Development of HIV-1-based vectors was first achieved by utilizing the abilities of lentiviral vectors of infecting post-mitotic cells. The design of other retrovirus-based vectors, such as self-inactivating MoMLV vectors, can be directly transferred to lentiviral vectors. In the most recent generations of HIV-1-based vectors, all accessory genes and the regulatory tat gene are deleted (Osten et al., 2007). In the self-inactivating (SIN) expression vector, the U3 promoter region is deleted from the 3'LTR. This region was copied to the $5^{\prime}$ end of dsDNA during reverse-transcription. Therefore, SIN modification results in transcriptional inactivation of the integrated provirus, which allows for the restriction of the likelihood of recombination with latent retroviral sequences and mobilization of latent retroviral sequences in the host cell genome (Osten et al., 2007).

Tropism of lentiviral vectors depends on the type of envelope protein used for viral production (Escors and Brecpot, 2010). The use of heterologous Env proteins is called pseudo typing. The most widely used Env protein is "vesicular stomatitis virus glycoprotein (VSV-G)". This protein permits the virus high titration values via ultracentrifugation and a wide tropism (VSV-G binds to suitable phospholipid component of plasma membrane) (Osten et al., 2007).

Lentiviral vectors do not require degradation of the nuclear membrane for integration. Lentiviruses that are encoded with the Gag matrix protein integrase enzyme and vpr protein 
interact with the nuclear import mechanism of the target cell and manage active transport of pre-integration complex via nucleopores. This characteristic enables transfection to nondividing cells and, in conclusion, "complex retroviruses" become suitable for use as gene transfer vectors, particularly for post-mitotic cells like neurons (Howarth et al., 2010). Receptors have been defined for many retroviruses. The best-characterized example is CD4 molecule, which serves as a receptor for lentiviruses including HIV (Freed, 1997).

Frequently, pseudotype lentiviral vectors are formed by "vesicular stomatitis virus" envelope (VSV-G). VSV-Gis a glycoprotein that interacts with the phospholipid component of a number of receptors or cell membrane. VSV-G offers large host-cell range and high vector particle stability, which are attractive characteristics for ex vivo gene modification. However, restriction of infection to specific cells, which is called transduction altargeting, is effective and reliable and critical in the case of in vivo gene delivery. In addition, it is the key to increase therapeutic effectiveness, reduce adverse effects, and reducing the required amount of the vector. Two methods can be used to achieve this: (a) benefiting from natural characteristics of existing viral proteins, (b) using gene engineering to continue, stop or increase the original tropism of the vector (Escors and Brecpot, 2010).

Recently-developed lentiviral gene transfer systems have many characteristics of retroviral systems. A viral genome integrates with host chromosomes, and genes that are desired to remain permanently are placed (Yi et al., 2011).

\subsubsection{Non-viral gene delivery systems}

The basic concept underlying gene therapy is that it develops gene expression to specific cells in order to treat human diseases or for transfer of genetic material to inhibit the production of a target protein (He et al., 2010).

In theory, viral carriers can achieve rapid transfection of foreign material spliced to viral genome with high transfection ratio. However, many studies that used viral vectors reported unsatisfactory results, due to the immunologic and oncogenic adverse effects of these vectors. On the other hand, non-viral vectors have many advantages, such as easy of fabrication, cell/tissue targeting, and low immune response. The biggest disadvantage of non-viral vectors in clinical use is low transfection efficiency (He et al., 2010). Therefore, the most important points that should be taken into account in gene therapy are the introduction of the gene into the cell and increasing transfection efficiency. Using transfection, cells are induced for the production of chemicals used in pharmacological arrangements like hormone replacement or specific protein production (Godbey and Mikos, 2001). Naked DNA molecules cannot effectively enter to the cell due to their hydrophilic structures and large size resulting from negatively-charged phosphate groups. In addition, they are easily fragmented by nuclease enzymes. Therefore, the biggest difficulty in gene therapy is the development of physical methods to ensure gene transfer to target cells of the gene delivery vectors and delivered gene (Al-Dosari and Gao, 2009).

As seen in Figure 4, natural and synthetic polymers are used to prepare non-viral gene delivery systems. Compared to viral delivery systems, non-viral carriers are less toxic and 
immunogenic. Another advantage of non-viral vectors is ease-of-production (DeLaporte et al., 2006). Much of the previous research found that non-viral systems were less effective than viral systems. However, in gene therapy conducted with physical methods, the effectiveness of gene transfection and therapy increased, and the duration of gene expression was extended significantly in clinical terms (Al-Dosari and Gao, 2009).

(a)

(b)

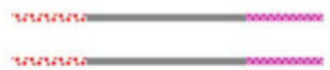

(c)

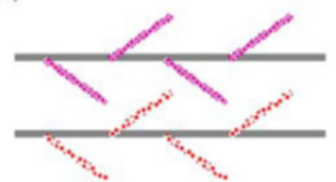

DNA

Vector backbone

Environmental

Interactions

Intracellular

Trafficking (d)

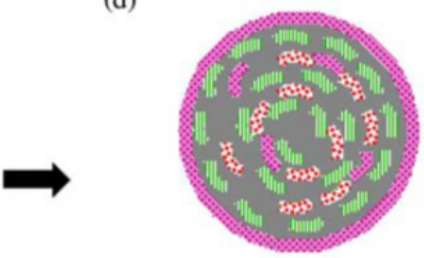

(e)
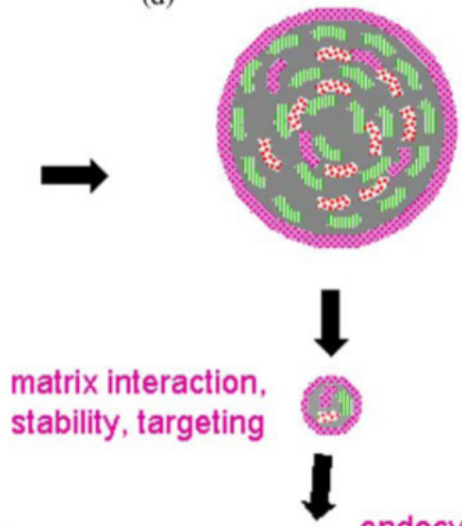
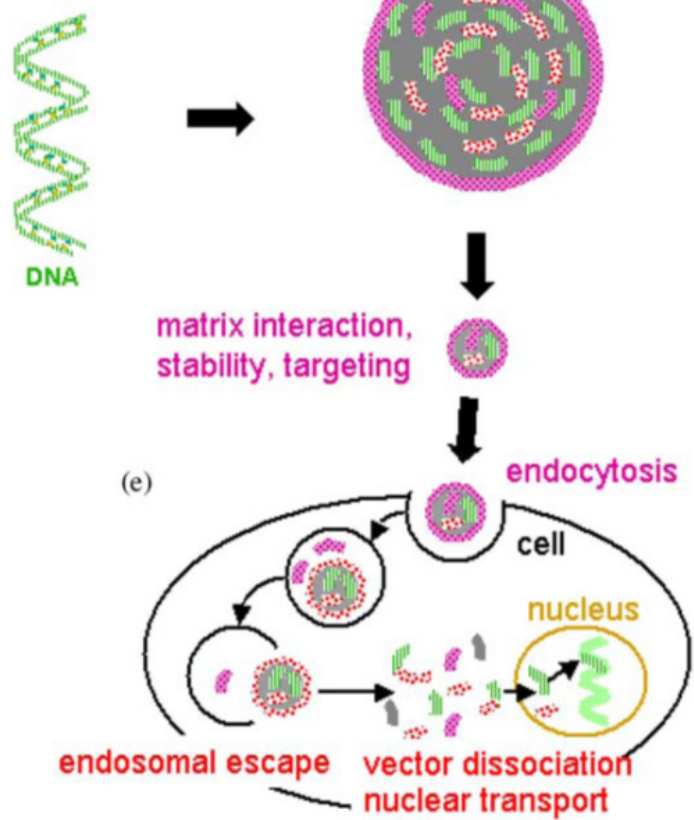

Figure 4. Modular design of non-viral vectors schematic. Modules associated with vector design are: vector backbone (grey), functional groups for regulating environmental interactions (purple), and intracellular trafficking (red). The vector backbone, typically containing polymers, lipids, or polysaccharides, is designed for DNA binding and complexation, which can protect against nuclease degradation, create a small, less negatively charged particle that can be internalized by cells, and facilitate some intracellular trafficking. The function of the vector backbone is being augmented by the attachment of groups that address the extracellular and intracellular barriers. The environmental functional groups can serve to limit interactions with serum components, promote specific cell binding or tissue targeting, or facilitate interactions with the extracellular matrix or biomaterials. The intracellular functional groups aim to enhance nuclear accumulation of the DNA either by facilitating endosomal escape, movement along the cytoskeleton, or nuclear pore trafficking. The individual modules can be assembled in different ways (a - c) for complexation with DNA (green), which may affect the structure and function of the resulting non-viral vector. (d) Schematic illustrating the distribution of the modules and DNA throughout the vector cross section, with the desired organization of functional groups regulating the environmental interactions presented primarily on the exterior and the groups for intracellular trafficking protected within the vector interior for activity following internalization. (e) Vectors are internalized by endocytosis and must subsequently escape the endosome for transport to the nucleus. Additionally, the modular components must dissociate from the DNA to allow for transcription (DeLaporte et al., 2006). 
A number of barriers need to be overcome in order to increase the effectiveness of non-viral vectors in humans. These barriers are classified as production/formulation/storage; extracellular barriers; and intracellular barriers (Davis, 2002). Anatomic barriers are extracellular matrixes coating the cells, which prevent direct transport of macromolecules to target cells through epithelium and endothelial cell sequences. Phagocytes like Kupffer cells in the liver and residential macrophages in the spleen are responsible for theclearance of DNA-loaded colloidal particles in blood circulation. Similarly, nucleases found in blood and extracellular matrix cause free and unprotected nucleic acids to be rapidly inactivated following systemic application. The most critical barrier to effective DNA transfection was regarded as the transition of plasma membrane. Typically, naked nucleic acids cannot cross cell membrane by cellular uptake mechanisms such as endocytosis, pinocytosis, and phagocytosis without application of physical methods or being loaded to a carrier (Al-Dosari and Gao, 2009). There is a substantial body of research on developing effective physical, chemical, and biological systems to deliver transgenes into the cell to provide appropriate expression (Gao et al., 2007). Physical approaches, including electroporation, gene gun, ultrasound, and hydrodynamic delivery are based on the application of a force to increase the permeability of the cell membrane and promote intracellular gene transfer. In chemical applications, synthetic or natural carriers are used to transport transgenes to the cells. An ideal gene delivery system should meet 3 criteria: The carriers should protect the transgene from nuclease enzymes inside intracellular matrixes; should transport the transgene from plasma membrane to the target cell nucleus; and should not cause any toxic effect (Gao et al., 2007).

Two of the most important advantages of synthetic carriers are that they do not display immunogenicity, and large-scale production is easy. Furthermore, the size of the gene to be delivered does not reduce efficiency of these systems. Even mammalian artificial chromosomes of 60 megabase were successfully transfected by these types of carriers (Schatzlein, 2003).

Non-viral vectors can trigger an inflammatory response, since they do not provide a specific recognition; they are much less dangerous than viral vectors in terms of antigen specific immune response. However, although non-viral vectors seem to be more suitable, there are some important points to be contemplated in vector design. Non-viral vectors should be designed according to specific cell targeting; cellular uptake and cellular release should be optimized; and potential immune response should be minimized (Conwell and Huang, 2005; Mrsny, 2005).

\subsubsection{Physical methods}

\section{Gene Gun}

Delivery with gene gun method is also termed ballistic DNA delivery or DNA-coated particle bombardment, and was first used for gene transfer to plants in 1987 (Al-Dosari and Gao, 2009; Lin et al., 2000). This method is based on the principle of delivery of DNA-coated heavy metal particles by crossing them from target tissue at a certain speed. The particles achieve sufficient speed due to a pressurized inert gas (generally helium). Generally, gold, tungsten or silver microparticles were used as the gene carrier (Mhashilkar et al., 2001; Al- 
Dosari and Gao, 2009; Miyazaki et al., 2006). Momentum allows penetration of these particles to a few millimeters of the tissue and then cellular DNA release (Gao et al., 2007). These particles typically have a diameter of $1 \mu \mathrm{m}$. Due to its small size, the particle easily penetrates the cell membrane and can transport DNA into the cell. At this point, DNA separates from the carrier particle and can be expressed (Lin et al., 2000).

Gas pressure, particle size and dose frequency are critical factors in determining the degree of tissue damage and penetration effectiveness of the application (Al-Dosari and Gao, 2009).

Gene-gun-based gene transfer is a widely tested method for intramuscular, intradermal and intratumoral genetic immunization (Miyazaki et al., 2006). Numerous animal tests and clinical trials indicated that this method caused a greater immune response than microinjection, even in low doses (Al-Dosari and Gao).

The advantages of gene gun over other in vivo gene delivery systems are as follows: [1] It does not use toxic chemicals or complex biological systems, [2] delivery is achieved without the need for a receptor, [3] DNA fragments of various sizes, including large ones, are transported, [4] there is no need to introduce foreign DNA or protein, [5] it has high repeatability, [6] production of heavy metal particles is easy (Lin et al., 2000). However, in this method, gene expression is short-term and low (Mhashilkar et al., 2001).

\section{Electroporation}

The future of gene therapy requires the advancement of effective and non-toxic polynucleotide gene delivery mechanisms. Electroporation includes controlled electric application to increase cell permeability. Electroporation was first developed in 1960s, with studies on the degradation of cell membrane with electric induction. Neumann et al., first reported transfection of eukaryotic culture cells through electroporation in 1982. In many subsequent studies, transfection was performed on animal and plant cells via electroporation (Al-Dosari and Gao, 2009; Somiari et al., 2000).

Electroporation introduces foreign genes into the cell by electric pulses. In this method, pores are formed on the membrane surface to enable the DNA to enter the cell. Pore formation occurs very rapidly, in approximately 10 nanoseconds. The size of the electric pore is estimated to be smaller than $10 \mathrm{~nm}$ radius. If the molecule is smaller than the pore size (as in oligonucleotides and chemical compounds), it can be transferred to the cell cytosol through diffusion (Somiari et al., 2000; Miyazaki et al., 2006). In addition to passive diffusion, loaded molecules and ions can be transported from the membrane via electrophoretic and electro-osmotic means via the effect of electric regions. The pores close following cellular uptake of DNA. In recent years, in vivo electroporation became common in nucleic acid vaccines and improving non-viral gene therapy. There are critical steps defined for transportation via in vivo gene delivery with electroporation. The first step is fusing together, attachment or proximity between the cell membrane and nucleic acid. The second step is adding nucleic acid to the membrane, which is immediately followed by the third step, involving the delivery of nucleic acid from the membrane. The delivery procedure results in decreased pore size and permeability (Somiari et al., 200). 
In vivo electroporation technique is a generally reliable method that is more efficient than other non-viral systems. When the parameters are optimized, this technique is equally effective as viral vectors. In addition to local injection and electroporation, it was previously shown that in vivo electroporation can be applied in localized form after the injection of plasmid DNA (Al-Dosari and Gao, 2009).

There are some difficulties in in vivo applications of electroporation. There is an effectiveness region of approximately $1 \mathrm{~cm}$ between the electrodes, and this makes transfection of the cells in large regions of the tissues difficult. It is difficult to use in internal organs, and surgery is required to implant electrodes. High temperature due to high voltage application can cause irreversible tissue damage. The application of high voltage to cells can affect the stability of genomic DNA (Al-Dosari and Gao, 2009; Gao et al., 2007).

\section{Ultrasound}

Ultrasound has many clinical advantages as a gene delivery system, due its easy and reliable procedure (Pitt et al., 2004; Ferrara, 2010). In recent years, it was found that microbubbles applied by ultrasound increased gene expression. Microbubbles or ultrasound contrast agents decrease cavitation threshold with ultrasound energy. In many applications, perfluoropropane-loaded albumin microbubbles (Mallinckrodt, San Diego, USA) were used. The microbubbles were modified with plasmid DNA before the injection and then ultrasound was applied (Niidome and Huang, 2002).

Ultrasound has been used for therapeutic purposes for more than a half century, even before the use of ultrasound for diagnosis and imaging purposes. In diagnostic use, low-intensity ultrasound is used to prevent energy accumulation in tissue causing biological effects. Conversely, therapeutic applications are based on the principle of intensification of ultrasound energy within the tissue (Frenkel, 2008).

After the discovery of the applicability of ultrasound to gene therapy at the cellular and tissue level, this was taken one step further with physical methods. Reporter gene expression, where naked DNA is used, advanced for10 times thanks to this discovery. The transfection efficiency of this system is based on frequency, time of ultrasound treatment, the plasmid DNA mount used, etc. In conclusion, size and local concentration of DNA plays an important role in the effectiveness of transfection (Gao et al., 2007).

\section{Hydrodynamic therapy}

Hydrodynamic gene delivery is based on the principle of understanding the characteristics and structure of capillaries, an understanding of the dynamic characteristics of the fluids passing through blood veins (Suda and Liu, 2007). Hydrodynamic gene delivery uses the hydrodynamic pressure created by the injection of the large volume of DNA solution with blood pressure inside veins (Suda and Liu, 2007), and thus the permeability of the capillary endothelium increases and pores form in the plasma membrane encircling parenchyma cells (Gao et al., 2007; Suda and Liu, 2007). DNA or other related molecules can reach the cell from these pores. Membrane pores are later closed, and these molecules are retained within the cell. In hydrodynamic gene therapy, the principle reason for targeting parenchyma cells 
is that capillary endothelium and parenchyma cells are closely related, and when the endothelial barrier is breached, this allows DNA to easily reach parenchyma cells. In addition, the capillary's thin wall structure has a stretchable and easily fragmented structure (Suda and Liu, 2007). The effectiveness of hydrodynamic procedure depends on capillary structure, the structure of the cells encircling capillaries, and the hydrodynamic force applied (Gao et al., 2007; Suda and Liu, 2007).

\subsubsection{Chemical methods}

\section{Polymers}

Polymers are long-chained structures composed of small spliced molecules called monomers. Polymers that are composed of a repeated monomer are called homopolymers, while those composed of two monomers are called copolymers. Natural and synthetic polymers are used in drug delivery systems. Biodegradable and non-biodegradable polymers are used according to the type of controlled release mechanism. Biodegradable polymers are non-water soluble, and undergo chemical or physical change in biologic environments (Tuncay and Calis, 1999). Polyamides, dextran, and chitosan are examples of biodegradable polymers. On the other hand, non-biodegradable polymers are not degraded in biological environments; hydrophilic polymers are hydrogels, which are non-water soluble and swell in water, while hydrophobic polymers are non-water soluble and do not swell. Examples of hydrophilic hydrogel polymers include polyvinylalcohol, polyvinylacetate, polyethyleneglycol, polyacrylic acid, polyhydroxyethyl methacrylate, and polymethacrylic acid. Examples of hydrophobic polymers include silicones, and polyethylene vinyl acetate. Polymers such as ethyl cellulose (EC), hydroxypropyl methyl cellulose (HPMC), cellulose acetate phthalate (CAP), and eudragit derivatives are commonly used in controlled release systems (Tuncay and Calis, 1999).

Selection and design of a polymer is difficult due to structural differences; achieving the desired chemical, mechanical, and biological functions requires understanding of the characteristics and surface of a polymer. For polymer selection, in addition to its physicochemical characteristics, characterization of extensive biochemical characteristics and preclinical tests are required to demonstrate its reliability (Pillai and Panchagnula, 2001).

The physical properties of a polymer carrier, such as hydrophilicity, surface charge, permeability and diffusibility, biocompatibility with tissue and blood (Pillai and Panchagnula, 2001).

For successful delivery, polymers should package DNA in small sizes. Thus, extracellular and intracellular stability of DNA is increased; cellular uptake by endocytosisis enabled; and, by transporting it to the nucleus, the active form of DNA can be released within the nucleus (Mrsny, 2005).

Polymeric gene transporters deliver genetic material through electrostatic interactions with nano-sized polyplexes for gene therapy (Kim et al., 2011).

A synthetic gene delivery system: [1] should protect the negatively-charged phosphate DNA skeleton against anionic cell surface from load repulsion, [2] should be condensed to suitable 
length intervals of DNA with a macromolecular structure for cellular internalization (condensing at nanometer size for receptor compatible endocytosis or condensing at micrometer size for phagocytosis); and [3] should protect DNA from all extracellular and intracellular nuclease degradations. To meet this need, researchers concentrated on three strategies: electrostatic interaction, encapsulation, and adsorption (Wong et al., 2007).

\section{Electrostatic interaction}

The majority of polymeric vectors presently in use form complexes with negatively charged DNA by electrostatic interaction. All polymers have amino groups. At adequate nitrogenphosphate ratio, the polymer and the DNA form nanocomplexes, which allows both cellular DNA uptake and also protects the DNA from nuclease enzyme (Wong et al., 2007).

\section{Encapsulation}

An alternative to electrostatic condensation of DNA is encapsulation of DNA with a biodegradable polymer. Polymers that have an ester linkage in their structures (like polyesters) are hydrolytically degraded to short oligomeric and monomeric compounds, which are more easily discharged from the body. Furthermore, the degradation mechanism and DNA release can be controlled by changing the physicochemical characteristics and composition of the polymer. DNA is protected from enzymatic degradation by encapsulation (Wong et al., 2007) (Figure 5).
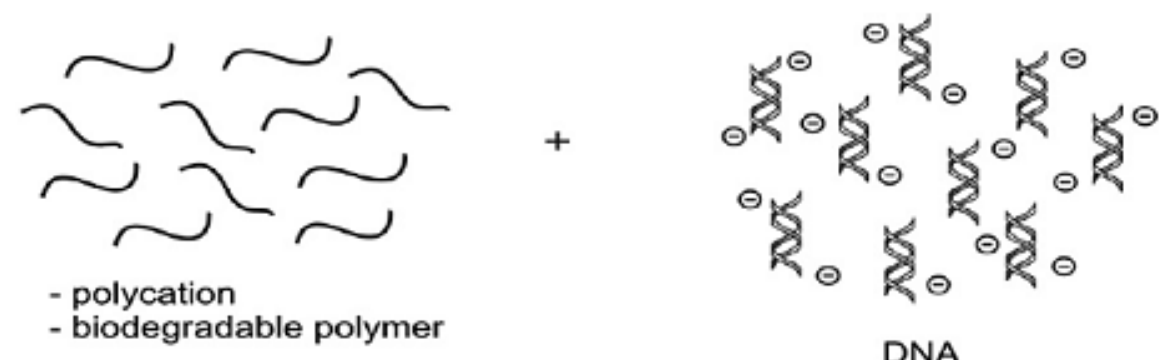

- biodegradable polymer

DNA

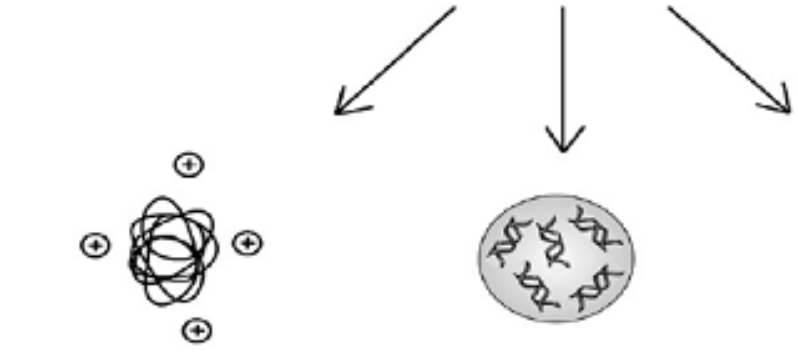

electrostatic interaction encapsulation

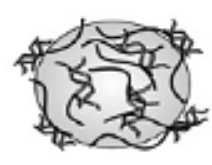

adsorption

Figure 5. Gene packaging - The three main strategies employed to package DNA are via (1) electrostatic interaction, (2) encapsulation within or (3) adsorption onto biodegradable nano- or microspheres (Wong et al., 2007). 


\section{Adsorption}

Alternative DNA packaging methods, such as adsorption techniques, were developed to overcome the limitations of encapsulation methods. DNA adsorption represents the combination of the above mention two models. In this method, DNA is conjugated to the surface of biodegradable cationic particles, or is electrostatically adsorbed due its negative charge. This method prevents the DNA from being exposed to heavy encapsulation conditions, and also increases rapid release DNA (Wong et al., 2007).

Adsorption methods attracted much research interest, as it does not cause immunogenicity, does not require the integration of exogenous genes to host chromosomes, is a simple and cheap method, and is suitable for effective gene delivery. However, low transfection efficiency, some cytotoxic effects, and in vivo instabilities are still significant restricting factors in gene therapy. One of the strategies to solve these problems is the synthesis of biologically degradable polymers (Kim et al., 2011).

\section{Liposomes}

Liposomes are colloidal drug delivery systems. They are biologic membrane-like sacs in sphere form, formed by one or more lipid layers, and include an aqueous phase. The phospholipid phase consists of principle components like aqueous phase and cholesterol. Liposomes are classified according to the number of layers they contain. The advantages of liposomes include effectiveness at small doses, extended dosing interval, and ideal transport for active substances with a short half-life. Cellular uptake mechanism of active substances in liposomes can be categorized as endocytosis, combination by melting, and adsorption. Liposomes are used for application of carcinogenic, antifungal, antiparasitic, antiviral, and anti-inflammatory drugs, hormones, DNAs, and cosmetics (Bogdansky, 1990).

Cationic lipids, which are used for the entrance of plasmid DNAs in the cell, have been studied for more than 20 years. During this period, many cationic liposome formulations were developed, and were tested as nucleic acid transported in animals and human with phase I and phase II studies. When compared to other gene delivery systems such as viral vectors and transfection agents, cationic liposome delivery systems are more easily formulated and cause no biological damage like viral vectors (Dass and Choong, 2006). Critical parameters that determine the behavior of liposomes in in vitro and in vivo conditions include size, number of layers, and surface charge. Unilamellar or mutilamellar vesicles can be produced depending on the preparation method; the diameter of these vesicle ranges from $25 \mathrm{~nm}$ to $50 \mu \mathrm{m}$ (Bogdansky, 1990).

Liposomes are divided into three categories according to their charges: cationic, anionic, or neutral. Compared to viral vectors, liposome delivery systems are non-pathogenic and nonimmunogenic, with easy of preparation. However, the most important disadvantage of liposomes is short gene expression time and low transgene expression level. Since liposomes are generally non-toxic and non-immunogenic structures, they are considered reliable carriers for gene therapy. Liposome-based gene delivery was first reported by Felgner (1987). In the 1990s, liposomes were used in numerous cationic lipid gene therapy tests. 
Liposomes are not as effective as viral vectors in in vivo gene delivery, and it is generally difficult for them to transfect targeted gene to selected cells (Miyazaki et al., 2006).

\section{Dendrimers}

Dendrimers were first defined in the late 1970s and early 1980s. Dendrimers consist of symmetrical branches projecting from a central core (Dufes et al., 2005; Genc, 2008; Bulut and Akar, 2012). Dendrimers are 10-200 Angstrom in diameter; they are repeating, branched, large spherical molecules, and have functional groups on their surface that can be used as a building block to connect many biological materials (Ward and Baker, 2008). These functional groups determine the variability of dendrimers, whereas branching provides the growth of the structure (Dufes et al., 2005; Bulut and Akar, 2012). As seen in Figure 6, the most important advantages of dendrimers are conjugation of a large number of different molecules on the dendrimer surface, and the conjugation of molecules that will be used for diagnosis and treatment (Ward and Baker, 2008; Dufes et al., 2005).
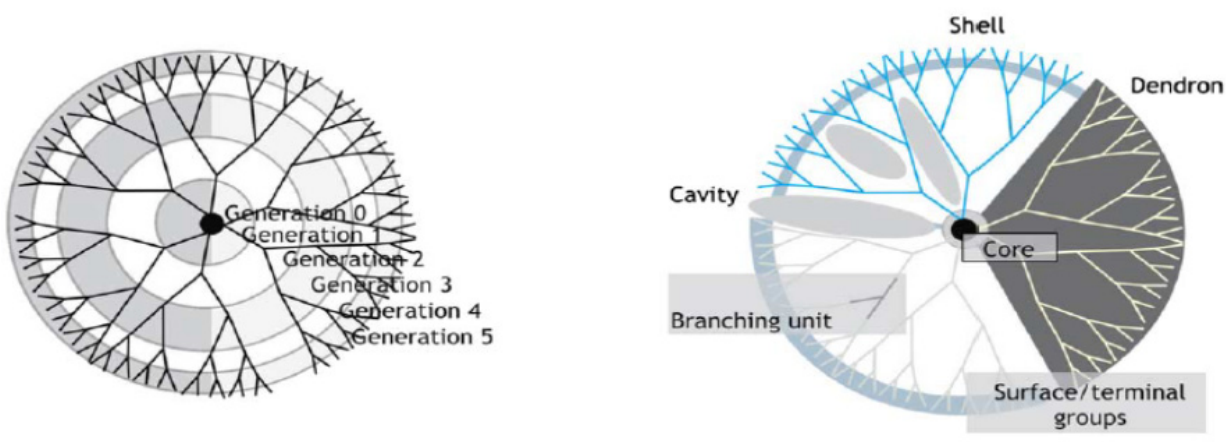

Figure 6. Dendrimer structure. The stepwise synthesis of dendrimers means that they have a well defined hierarchical structure. This hypothetical dendrimer is based on a core with three covalent root attachment points but other common cores have di- or tetracovalent cores. The valency of the core dictates the number of linked dendrons and the overall symmetry of the molecule. The dendrons are synthesised by covalent coupling of the branch units. For each additional layer or generation that is being added to the structure the reaction sequence is repeated. In this case the units have two new branching points at which additional units can be attached. (The generation count is not always consistent: normally generation 0 refers to the core while sometimes it is used to describe the dendrimer after the first reaction cycle.) The number of branching points, branching angles, and the length of the branching units determine to what extent each generation increases molecular volume vs. surface area. For the higher generations the density of the terminal groups reaches a point where for steric reasons no groups can be added (starburst effect). Dendrimers of higher generation also have a typical molecular density profile under favourable conditions; the high peripheral molecular density establishes a steric outer shell and the lower density at the centre creates cavities which can accommodate guest molecules. (Dufes et al., 2005).

The degree of polymerization of dendrimers is represented by the number of generations (G) of repeated branching cycles during synthesis. Generation number can be easily identified by calculating number of branching points from the core to the outer surface. 
The number of branching points increases proportional to dendrimer growth. For example, a dendrimer with no connection points is termed zero generation (G-0) (Bulut and Akar, 2012).

Equal layer groups of dendrimers with an equal number of layers are highly suitable for use in drug delivery due to their perfect encapsulation characteristics and highly controllable chemical behaviors. Drugs can be encapsulated in a dendrimer or can electrostatically or covalently connect to functional groups on the surface (Ward and Baker, 2008; Bulut and Akar, 2012). On the other hand, targeted drug delivery can be achieved by covalent conjugation of the targeting agent to functional groups of dendrimers (Ward and Baker, 2008).

Although the toxicity of dendrimers that form complexes with DNA generally decreases, toxicity and cellular effects of free dendrimers should be taken into account (Dufes et al., 2005).

\section{Cationic lipid compatible systems}

Felgner et al. pioneered cationic lipid-based gene transfer (Felgner et al., 1987; Al-Dosari and Gao, 2009). Today, hundreds of lipids have been developed and tested for gene transfer. Lipid-based systems consist of a positively-charged hydrophilic head and hydrophobic tail structure.

Most common hydrophilic head groups are primary, secondary, tertiary amines, or quaternary amine salts. These positively charged sections connect to negatively charged phosphate groups in nucleic acid. The hydrophobic tail consists of aliphatic chain, cholesterol, or steroid rings. The connection region between hydrophilic and hydrophobic sections consists of the bonds that affect biodegradability ratio, such as ether, ester, carbamate, or amid (Al-Dosari and Gao, 2009; Uyechi-O’Brian and Szoka, 2003).

Each lipid has a different sized head group and hydrocarbon tail length, and these characteristics have a significant impact on the formation of DNA-lipid complexes and intake by the cell (Balasz and Godbey, 2010). The transfection effectiveness of cationic lipids varies according to the structure of the lipid and the charge ratio used to form the DNAlipid complex. Compared to negatively-charged DNA, a positively-charged lipid spontaneously forms structures called lipoplexes. In lipoplex structure, the DNA molecule is encircled by positively-charged lipids, which protect it from extracellular and intracellular nucleases. In addition, due to their positive charges, lipoplexes electrostatically interact with the cell membrane and thus cellular uptake is achieved (Al-Dosari and Gao, 2009; UyechiO'Brian and Szoka, 2003).

Cationic lipids such as $\mathrm{N}$-[1-(2,3-dioliloksi)propyl]-N,N,N-trimethlammoniumchloride (DOTMA), [1,2-bis(oliloxy)-3-(trimethlamonnium) propane] (DOTAP), $3 \beta\left[\mathrm{N}-\left(\mathrm{N}_{-} \mathrm{N}_{-}-\right.\right.$ dimethlaminoethane)-carbamile] cholesterol (DCChol) and dioctadecylamidolysisspermine are used to prepare lipid carriers (Balasz and Godbey, 2010). 
The aim of cationic lipid-based gene therapy is to transport plasmid DNA to the cell and thus to achieve the desired protein/peptide transcription and translation. DNA-lipid complex is internalized into the cell through the vesicular path, which is followed by the release of cell cytoplasm of DNA from the lipoplex. Some fractions of the released DNA are transported to the nucleus (Uyechi-O'Brian and Szoka, 2003). Strong DNA-cationic lipid complexes alone are insufficient for gene transfer; however, DNA release before or after transport to the nucleus is critically important (Khalid et al., 2008). Lipid-cationic lipid ratio, cationic lipid-DNA ratio and DNA mount presented to the cell are important parameters that significantly influence transfection efficiency (Hofland et al., 1996).

Although gene expression is provided by cationic lipids, for clinical benefit, the effectiveness, expression time and degree of gene transfer were not found to be adequate. The most significant disadvantages of cationic lipids are structural instability and heterogeneity, inactivation in blood, low effectiveness, and weak targetability when compared to other systems (Navarro et al., 2008).

Cationic lipid-based transfection systems are easy to prepare and reliable; they also have low immunogenicity, and there is no need to classify the size of DNA which will be transported (Mortimer et al., 1999). Many cationic lipid-DNA based systems are effectively taken up by endocytosis; however, the mechanism of DNA release from endosome and translocation to the nucleus are not well known (Mortimer et al., 1999).

\subsection{The use of gene delivery systems in treatment}

\subsubsection{Cancer}

Any change in the structure of genes that cause diseases is called mutation. In a mutated cell, variations occur in the sequence of nucleotides. As a result, chemical substances that are produced under the control of the gene have abnormal structure and function. If the gene is associated with the growth, differentiation, or division of cells, then the mutation might cause cancer (Wang et al., 2012). There are two main groups of genes responsible for formation of cancer. These are oncogenes and tumor suppressor genes. Oncogenes are mutated versions of normal genes that are responsible for controlling cell growth. After mutation, they compel the cell to become a cancer cell. There are more than 100 known oncogenes, most of which were identified in human tumors. The "ras gene" is the most widely known and most commonly studied of these genes. After mutation, the ras gene initiates division and cancer formation in cells with the "ras protein" it forms (Luo et al., 2012).

Tumor suppressor genes normally inhibit cell division and tumor development in cells. The mutated forms of these genes cannot inhibit cell division, and instead cause tumor formation. Among these genes, the RB and P53 genes are the most widely known. These genes show their effect with p-RB protein and P53 protein, which are produced under their control. Normally, these proteins inhibit DNA replication and cell division, and regulate apoptosis. If the created genes are mutated, these proteins lose their activity and 
uncontrolled cell division occurs, which results in cancer development. It was indicated that RB protein was inactive in approximately $40 \%$ of human cancers. Similarly, it was found that P53 gene had an abnormal structure in 50\% of all tumors (Morandell \& Yaffe, 2012).

Gene therapy is based on the principle of replacing damaged genes in diseased cells with corresponding healthy genes that were prepared outside the body. This approach is the most difficult type of gene therapy. Successful treatment involves healthy genes, which are prepared out side the body, targeting specific cells in the body via certain transporters (carriers). Current technology has simplified the process of preparing healthy genes. However, the subsequent process of targeting cells remains imperfect (Lagisetty \& Morgan, 2012).

In addition to the correction of defective genes, gene therapy includes other treatment principles. In a different method, cancer patients were injected "cytosinedeaminase" enzyme, which is not normally produced in humans, via viral carriers, thereby allowing this enzyme to be synthesized in tumor cells (Logue \& Morrison, 2012). The drug 5-FC is injected at high doses with no adverse effects; this is converted to 5-FU in tumor cells by cytosine deaminase enzyme. Using this method, effective levels of drug activation are achieved without damaging healthy cells.

In a similar method, carrier viruses injected with "thymidinekinase enzyme gene" found in the herpes simplex viruses reach cancer cells and produce thymidine kinase enzyme in these cells. This enzyme enables the drug ganciclovir, which has no adverse effects on normal body cells, to be active in tumor cells and destroy cancer cells (Etienne-Grimaldi et al., 2012)

Another treatment approach involves inhibition of oncogene proteins that were mentioned above. Oncogenes cause cancer formation via the proteins they control. If the production of these proteins is inhibited, their cancer-causing effects will be reduced. One example is the farnesyltransferase enzyme, which plays a role in the formation stages of RB protein. It was reported that new tumor formation was inhibited in patients who were injected with drugs that inhibited the function of this enzyme (Logue \& Morrison, 2012). Cancer treatment is the most studied application of gene therapy. A large number of genetic changes occur by the transformation of a normal cell to a neoplastic cell (El-Aneed, 2004).

Tumor suppressor genes eliminate cancer cells through apoptosis, whereas oncogenes accelerate proliferation. For this reason, apoptotic genes and anti-oncogenes are frequently used in cancer treatment. In addition to oncogenes and tumor suppressor genes, chemotherapy and gene therapy were combined via a 'suicide gene' strategy. A suicide gene encodes an enzyme that does not belong to mammals, and this enzyme converts non-toxic product into active cytotoxic metabolite in cancer cells. Tumor suppressor genes, antioncogenes, and suicide genes target cancer cells at the molecular level. Generally the genes that encode cytokine revive immune response to cancer cells (Wang et al., 2012). 
Transformation of a normal cell to a neoplastic cell occurs by multi-mutationary changes of the cells at the genetic level. Due to the complicated nature of cancer, cancer gene therapy involves many therapeutic strategies. These strategies can be analyzed according to two categories: immunogenic and molecular (El-Aneed, 2004).

\subsubsection{Immunologic approach}

The human immune system has two pathways to respond to foreign antigens. The first pathway includes antibodies secreted by B cells following activation of membrane immunoglobulin via (B cell receptor)-antigen connection. Antibodies are water-soluble proteins that move within the circulatory system to reach the target antigens. $\mathrm{T}$ cells, the second immune pathway, directly interact with antibodies without secreting antibody. T cells can produce multiple immune reactions, including cytotoxic effects (Lagisetty \& Morgan et al., 2012).

Cancer cells are immunogenic in nature with their cancer antigens, which are intracellular molecules. Therefore, cellular immunity (T-cell mediated), is more important than humoral immunity (B-cell mediated) (Restifo et al., 2012). Normal immune response is not sufficient to eliminate tumor cells. The ability of cancer cells evade immune-system responses is associated with secretion of immunosuppressive factors, down-regulation of antigen expression or MHC (major histocompatibility complex) molecules, and co-stimulation deficiency. One of the common genetic immunotherapy strategies includes transfer of immune-stimulating molecular genes like cytokines. Immune response is created by the activation of interleukin-12 production by tumor cells with numerous components of immune system, particularly including cytotoxic $\mathrm{T}$ lymphocytes and natural killer cells (McDonald et al., 2012).

Another immunologic approach involves in vitro manipulation of the antigen-presenting cells to produce active tumor antigens. Dendritic cells are the strongest antigen-presenting cells (El-Aneed, 2004). Application of antigen-encoding genes via direct vaccination might induce the desired immune response to cancer cells. When injected subcutaneously or intramuscularly, DNA enters local cells like fibroblast and myocytes, and antigen is subsequently produced and secreted. Antigen-presenting cells move towards lymphoid organs where new antigens will be caught and the desired immune response will be initiated (El-Aneed, 2004). Another method to increase the specificity of chemotherapy might be enzyme-encoding enzyme-activate prodrug therapy, which converts non-toxic products into specific and toxic metabolites (Altaner, 2008). This approach is known by many different names, including enzyme prodrug therapy in gene management; suicide gene therapy or enzyme prodrug therapy in virus management; and gene prodrug activation therapy (Scheier et al., 2012). All of these expressions define the same two-step treatment process. The first step involves the targeting and transport of the foreign enzyme to the tumor via various pathways. In the second step, non-toxic prodrug is applied and converted to active systolic metabolite. In in vivo approaches, expression of foreign genes does not occur in each cell of the target tumor. As a result, the active drug 
can enter the transduced cell and cell deaths take place, including the cells which do not express enzyme. This procedure is also termed the bystander effect or neighbor cell death effect. In addition, dead cells can induce host immune response due to T cells and NK cells. This therapeutically beneficial effect is known as the slight bystander effect. To increase the effectiveness of enzyme-activated product therapy, therapeutic genes in the vector should be expressed at a sufficiently high ratio to achieve effective prodrug transformation; it should remain as active as possible in order to support the killing mechanisms of the produced systolic metabolite, and the suicide gene should be specifically targeted only to the tumor. These arrangements will also reduce adverse effects (Altaner, 2008).

\subsubsection{Cardiovascular disorders}

Recombinant adenovirus is one of the most practical vectors for localized gene therapy. In addition, these vectors allow for tissue-specific delivery, and are suitable for re-targeting. Bispecific antibodies were used to coat adenovirus particles by connecting the antibody ligand on the cell receptor surface. Genetic modifications also include gene modifications for some viral coat proteins to change tropisms of adenoviruses (Ji et al., 2012).

Antiviral vectors are the most recently developed vectors; they are HIV-based and generally used for HIV treatment. These vectors have the ability to transfect non-dividing and dividing cells. The effectiveness and reliability of these cells are currently being analyzed (Kozarsky, 2001).

\subsubsection{Angiogenesis}

One exciting recent development is that blood circulation can be provided to ischemic tissues. The development of new blood veins was observed in the region of endothelial growth factor (VEGF) expression (Katz et al., 2012). Gene transfer has advantages for protein therapy, and the use of permanent VEGF is preferred. This method can be fulfilled by a single injection of gene-transfer vector; however, transgene expression of VEGF should last for a limited period, because long-term unregulated VEGF expression might cause abnormal angiogenesis. Therefore, rapid gene expression obtained from adenoviral and plasmid vectors are among the ideal vectors for local delivery of VEGF gene by direct injection (Kozarsky, 2001; Katz et al., 2012).

\subsubsection{Cardiac failure}

Treatment of cardiac failure by gene therapy is still limited, due to the need to determine suitable transgenes and develop effective gene delivery methods to the myocardium. Genes can be directly transported to the heart. However, if these genes have to be transported to larger regions, as in the case of cardiac failure, this method is not regarded as successful. Another method involves transporting gene delivery vectors to coronary veins via a catheter; however, some catheter materials inactivate recombinant adenovirus (Kozarsky, 2001; Kairouz et al., 2012). 


\subsection{Pulmonary disorders}

According to some authors, lungs are ideal organs for gene therapy. However, cystic fibrosis and $\alpha_{1}$-antitripsin deficiency are the only common disorders with known genetic background for which existing treatments are not curative. Although cystic fibrosis is the first target for pulmonary gene therapy, this method is used to treat many acquired diseases, mainly cancer (Davies et al., 2003).

After the discovery of CTFR in 1989, considerable new progress was made to improve gene transfer system for somatic gene therapy of cystic fibrosis. Cystic fibrosis affects approximately1 in 2500 newborns, which makes it the most common recessive genetic lethal disorder (Davies et al., 2003). Even a copy of a single normal SF gene is sufficient for normal function (Klink et al., 2004). Therefore, somatic gene therapy is relatively straightforward. The only requirement is to provide a cell affected by a gene which makes CFTR protein expression (94). CFTR is cAMP-regulated chloride channel in epithelium cells. Dysfunction of CFTR arises from production deficiency, problems in transport of apical membrane of the cell to the effective region, or due to functional defects (Davies et al., 2003). Air tract epithelium is the most important target among lung diseases that cause mortality and morbidity. In order to address this aim, many gene delivery systems were developed and their effectiveness in vitro and in vivo was analyzed.

The use of adenoviral vectors gave rise to safety concerns due to low transduction effectiveness and increasing inflammation in human lungs. Tests with cationic liposomes showed low toxicity and effectiveness (Klink et al., 2004).

Some individuals have to be overcome to perform gene therapy. Mucociliary clearance is the primary one of these individuals. Air tract secretions and the mucociliary clearance system act as significant barriers to exogenous gene transfer. The epithelia of a healthy respiratory system create a thin mucus layer required for normal cilia function; however, this layer inhibits gene transfer by cationic liposomes (Davies et al., 2003).

Similarly, a potential gene transfer agent has to overcome the barrier formed by secretions in order to enter the cell. The glycocalyx structure and endocytosis ratio of a cell were defined as the factors that restrict the effectiveness of gene transfer. A number of intracellular process, including endosomal system, cytoplasmic delivery, and the entrance to the nucleus serve as barriers to successful gene transfer. Similarly, host immune response causes problems in the application of gene transfer agents to lungs (Davies et al., 2003).

Adenovirus/CFTR was applied to the nasal epithelia of three patients to treat cystic fibrosis. No viral replication was observed, but mild inflammation was reported. PD returned to normal baseline values in all three patients (Davies et al., 2003). However, one of the problems of this vector system is that low expression levels of viral gene wastes leads to cytotoxic T-cell response that targets vector-containing cells. The fact that adenoviruses are generally air tract pathogens is a both advantageous and disadvantageous. Many potential 
receivers have circulating antibodies that reduce vector effectiveness and T-cell response. Most importantly, systemic application of adenoviral vectors causes acute and potentially life-threating inflammation responses due to activation of antigen-presenting cells (Klink et al., 2004). On the other hand, when compared to other gene delivery systems, lentiviral vectors are advantageous. Lentiviral vectors are integratable retrovirus derivatives with a high cloning capacity. When suitable regular sequences such as LCS (locus control sections) are used, stable and cell-specific expression can be achieved. When envelope proteins of pseudo-written lentiviral vectors are applied to apical surface with vesicular stomatitis virus, it is not possible to effectively transduce polarized air tract epithelia. Retroviral vectors, pseudo-written with apical membrane-connecting envelope proteins, are used to overcome this problem (Klink et al., 2004).

The first clinical trial of liposome-compatible CFTR gene transfer was reported in 1995. This method involves direct application of DC-Chol/DOPE/CFTR to the nasal epithelia of SF patients. No adverse clinical effects were observed, and nasal biopsy analyses showed no histologic change. Specific mRNA and plasmid DNA were found in five out of eight patients who received CFTR gene vector (Klink et al., 2004).

\section{Conclusion}

Current gene delivery systems are divided into three categories: viral-based, non-viral based, and combined hybrid systems. Viral gene delivery systems consist of viruses that are modified to be replication-deficient which can deliver genes to the target cells to provide expression. Although viral systems have many advantages, such as long-term expression, and the effectiveness and expression of therapeutic genes, they also have various disadvantages. These disadvantages include risks due to working with viruses, optimization problems when producing large batches, and problems of immunogenicity and toxicity. To date, adenoviruses, retroviruses, and lentiviruses have been used as viral gene carrier vectors. Non-viral systems include physical and chemical methods. In its broadest sense, this refers to delivery of the gene into the cell by applying a physical force in order to increase the permeability of the cell membrane. The most common physical methods are microinjection, electroporation, ultrasound, gene gun, and hydrodynamic delivery. Chemical methods use natural and synthetic compounds are to deliver the gene into the cell. The gene delivery system generally includes polymers, liposomes, dendrimers, and cationic lipids. Non-viral vectors have many advantages, such as easy of synthesis, effective targeting of cell/tissue, low immune response, and potential to use plasmid at desired molecular weight. The biggest disadvantage of non-viral vectors in clinical use is low transfection efficiency. A large number of viral and non-viral gene delivery systems have been developed. Both systems have many advantages and disadvantages. Hybrid systems were developed to combine the advantageous parts of both the individual systems, while minimizing their respective disadvantages, thereby producing a gene therapy with higher effectiveness and low toxicity. The biggest advantage of hybrid systems is that they are less toxic than viral systems, and are not as low-efficient as non-viral systems. 


\section{Author details}

Erdal Cevher * and Emre Şefik Çağlar

Department of Pharmaceutical Technology, Faculty of Pharmacy, Istanbul University, Beyazıt,

Istanbul, Turkey

Ali Demir Sezer

Department of Pharmaceutical Biotechnology, Faculty of Pharmacy, Marmara University,

Haydarpaşa, Istanbul, Turkey

\section{References}

Al-Dosari MS, Gao X. (2009). Nonviral Gene Delivery: Principles, Limitations, and Recent Progress. American Association of Pharmaceutical Scientist Journal; 11; 671-681.

Altaner C (2008). Prodrug cancer gene therapy. Cancer Letters; 270; 191-201.

Armendáriz-Borunda J, Bastidas-Ramírez BE, Sandoval-Rodríguez A, González-Cuevas J, Gómez-Meda B, García-Bañuelos J. (2011). Production of first generation adenoviral vectors for preclinical protocols: Amplification, purification and functional titration. Journal of Bioscience and Bioengineering; 112; 415-421.

Balazs DA, Godbey WT. (2010). Liposomes for Use in Gene Delivery. Journal of Drug Delivery; 2011; 1-12.

Bogdansky S. (1990). In Chasin M, Langer R(ed). Natural Polymers as Drug Delivery Systems. Biodagradable Polymers as Drug Delivery Systems. Marcel Dekker Inc, ABD. pp: 231-261.

Bulut MO, Akar E. (2012). Dendrimerlerin Önemi Ve Kullanım Alanları. Süleyman Demirel Üniversitesi Teknik Bilimler Dergisi: 1; 5-11.

Bupp K, Roth MJ. (2002). Altering Retroviral Tropism Using a Random-Display Envelope Library. Molecular Therapy; 5; 329-335.

Campos SK, Barry MA. (2007). Current Advances and Future Challenges in Adenoviral Vector Biology and Targeting. Current Gene Therapy: 7; 189-204.

Conwell CC, Huang L, In K. Taira, K. Kataoka, T. Niidome (ed). (2005). Recent Progress in Non-viral Gene Delivery. Non-viral Gene Therapy Gene Design and Delivery. SpringerVerlag Tokyo. Japan; pp: 3-11.

Coughlan L, Alba R, Parker A, Bradshaw AC, McNeish IA, Nicklin SA Baker AH. (2010). Tropism-Modification Strategies for Targeted Gene Delivery Using Adenoviral Vectors. Viruses: 2; 2290-2355.

Dass CR, Choong PFM. (2006). Selective gene delivery for cancer therapy using cationic liposomes: In vivo proof of applicability. Journal of Controlled Release; 113; 155-163.

Davies JC, Geddes DM, Alton EWFW. (2003). Pulmonary Gene Therapy. Pharmaceutical Gene Delivery Systems. Eastern Hemisphere Distribution, ABD. Chapter 14.

\footnotetext{
${ }^{*}$ Corresponding Author
} 
Davis ME. (2002). Non-viral gene delivery systems. Current Opinion in Biotechnology: 13: 128131

Dinh AT, Theofanous T, Mitragotri S. (2005). A Model for Intracellular Trafficking of Adenoviral Vectors. Biophysical Journal; 89; 1574-1588.

Dufes C, Uchegbu IF, Schatzlein AG. (2005). Dendrimers in gene delivery. Advanced Drug Delivery Reviews; 57; 2177- 2202.

El-Aneed A. (2004). An overview of current delivery systems in cancer gene therapy. Journal of Controlled Release; 94; 1- 14.

Escors D, Brecpot K. (2010). Lentiviral vectors in gene therapy: their current status and future potential. Archivum Immunologiae et Therapia Experimentalis;58;107-119.

Etienne-Grimaldi MC, Bennouna J, Formento JL, Douillard JY, Francoual M, Hennebelle I, Chatelut E, Francois E, Faroux R, El Hannani C, Jacob JH, Milano G. (2012). Multifactorial pharmacogenetic analysis in colorectal cancer patients receiving 5fluorouracil-based therapy together with cetuximab-irinotecan. British Journal of Clinical Pharmacology; 73; 776-785.

Felgner PL, Gadek TR, Holm M, Roman R, Chan HW, Wenz M, orthrop JP, Ringold GM, Danielsen M. (1987) Lipofection: a highly efficient, lipid-mediated DNAtransfection procedure. Proceedings of the National Academy of Sciences of U.S.A; 84; 74137417.

Ferrara KW. (2010). Driving delivery vehicles with ultrasound. Advanced Drug Delivery Reviews; 60: 1097-1102.

Freed EO. (1997). Retroviruses. Encyclopedia of Cancer:4; 167-172

Frenkel V. (2008). Ultrasound mediated delivery of drugs and genes to solid tumors Advanced Drug Delivery Reviews; 60: 1193-1208

Gao X, Kim KS, Liu D. (2007). Nonviral Gene Delivery: What We Know and What Is Next. American Association of Pharmaceutical Scientist Journal; 9: 92-104.

Genç R. (2008). Dendrimerler: Yeni Nesil Polimerik Ağaçlar. Katalizör: 1; 24-28.

Godbey WT, Mikos AG. (2001). Recent progress in gene delivery using non-viral transfer Complexes. Journal of Controlled Release: 72; 115-125.

Gönen Korkmaz C. (2008). Gen Transferi-Transfeksiyon: Prostat Kanseri STAMP2 Stabil Hücre Hattının Oluşturulması. DEÜ Tıp Fakültesi Dergisi; 22: 39 - 45.

He CH, Tabata Y, Gao JQ. (2010). Non-viral gene delivery carrier and its three-dimensional transfection system. International Journal of Pharmaceutics:386; 232-242.

Hofland HEJ, Shephard L, Sullivan SM. (1996). Formation of stable cationic lipid/DNA complexes for gene transfer. Proceedings of the National Academy of Sciences; 93: 73057309 .

Howarth JL, Lee YB, Uney JB, (2010). Using Viral Vectors as Gene Transfer Tools. Cell Biology Toxicology; 26; 1-20.

Hu WS, Pathak VK (2000). Design of Retroviral Vectors and Helper Cells for Gene Therapy. Pharmacological Rewievs; 52; 494-507. 
Smith JG, Wiethoff CM, Phoebe LS, Nemerow GR. (2010). Adenovirus. Current Topics in Microbiology and Immunology: 343; 195-224.

Ji J, Ji SY, Yang JA, He X, Yang XH, Ling WP, Chen XL. (2012). Ultrasound-targeted transfection of tissue-type plasminogen activator gene carried by albumin nanoparticles to dog myocardium to prevent thrombosis after heart mechanical valve replacement. International Journal of Nanomedicine;7;2911-2919.

Karlsson S, Papayannopoulou T, Schweiger S (1987). Retroviral-mediated transfer of genomic globin genes leads to regulated production of RNA and protein. Proceedings of the National Academy of Sciences; 84; 2411-2415.

Katz MG, Fargnoli AS, Pritchette LA, Bridges CR. (2012). Gene delivery technologies for cardiac applications. Gene Therapy;19;656-669.

Kairouz V, Lipskaia L, Hajjar RJ, Chemaly ER. (2012). Molecular targets in heart failure gene therapy: current controversies and translational perspectives. Annals of the New York Academy of Sciences; $1254 ; 42-50$.

Khalid S, Bond JB, Holyoake J, Hawtin RW, Sansom MSP (2008). DNA and lipid bilayers: self-assembly and insertion. Journal of the Royal Society Interface; 5; 241-250.

Khare R, Chen CY, Weaver EA, Barry MA. (2011). Advances and Future Challenges in Adenoviral Vector Pharmacology and Targeting. Current Gene Therapy: 11; 241-258.

Kim T, Rothmund T, Kissel T, Kim SW. (2011). Bioreducible polymers with cell penetrating and endosome buffering functionality for gene delivery systems. Journal of Controlled Release: 152; 110-119.

Kitamura T, Koshino Y, Shibata F, Oki T, Nakajima H, Nosaka T, Kumagai H. (2003). Retrovirus-mediated gene transfer and expression cloning: powerful tools in functional genomics. Experimental Hematology; 31;1007-1014.

Klink D, Schindelhauer D, Laner A, Tucker T, Bebok Z, Schwiebert EM, Boyd AC, Scholte BJ. (2004). Gene delivery systems-gene therapy vectors for cystic fibrosis. Journal of Cystic Fibrosis; 3; 203- 212.

Kozarsky KF (2001). Gene Therapy for Cardiovascular Disease. Current Opinion in Pharmacology 2001; 1; 197-202.

Lagisetty KH, Morgan RA. (2012). Cancer therapy with genetically-modified T cells for the treatment of melanoma. Journal of Gene Medicine; 14; 400-404.

Lin MTS, Pulkininen L, Uitto J, Yoon K. (2000). The Gene Gun: Current Applicationsin Cutaneous GeneTherapy. International Journal of Dermatology; 39; 161-170.

Logue JS, Morrison DK. (2012). Complexity in the signaling network: insights from the use of targeted inhibitors in cancer therapy. Genes $\mathcal{E}$ Development; 26; 641-650.

Luo Y, Kofod-Olsen E, Christensen R, Sørensen CB, Bolund L. (2012). Targeted genome editing by recombinant adeno-associated virus (rAAV) vectors for generating genetically modified pigs. Journal of Genetics and Genomics; 39; 269-274.

Majhen D, Ambriovic-Ristov A. (2006). Adenoviral vectors-How to use them in cancer gene therapy? Virus Research;119; 121-133. 
Mátrai J, Chuah MKL, Vanden Driessche T. (2009). Recent Advances in Lentiviral Vector Development and Applications. Molecular Therapy; 18: 477-490.

McDonald PC, Winum JY, Supuran CT, Dedhar S. (2012). Recent developments in targeting carbonic anhydrase IX for cancer therapeutics. Oncotarget; 3, 84-97.

Medina-Kauwe LK. (2003). Endocytosis of adenovirus and adenovirus capsid proteins. Advanced Drug Delivery Reviews; 55;1485-1496.

Mhashilkar A, Chada S, Roth JA, Ramesh R. (2001). Gene therapy; Therapeutic approaches and implications. Biotechnology Advances; 19; 279-297.

Miyazaki M, Obata Y, Abe K, Furusu A, Koji T, Tabata Y,Kohno S. (2006). Technological Advances in Peritoneal Dialysis Research: Gene Transfer Using Nonviral Delivery Systems. Peritoneal Dialysis International; 26; 633-640.

Miyazaki Y, Miyake A, Nomaguchi A, Adachi A. (2011). Structural dynamics of retroviral genome and the packaging. Frontiers in Microbiology; 2; 2-9.

Mortimer I, Tam P, MacLachlan I, Graham RW, Saravolac EG, Joshi PB. (1999). Cationic lipid-mediated transfection of cells in culture requires mitotic activity. Gene Therapy; 6; 403-411.

Mrsny R. (2005). In Amiji MM (ed). Tissue-and Cell-Specific Targeting for the Delivery of Genetic Information. Polimeric Gene Delivery. CRC PRESS, USA. pp: 1-30.

Muzzonigro TS, Ghivizzani SC, Robbins PD, Evans CH. 1999. The Role of Gene Therapy: Fact or Fiction? Clinics in Sports Medicine; 18; 223-237.

Navarro J, Risco, Toschi M,Schattman G. (2008). Gene Therapy and Intracytoplasmatic Sperm Injection (ICSI) - A Review. Placenta; 29; S193-S199.

Niidome T, Huang L. (2002). Gene Therapy Progress and Prospects: Nonviral vectors. Gene Therapy; $9 ; 1647-1652$.

Olsen JC, Swanstorm R. (1985). A New Pathway in the Generation of Defective Retrovirus DNA. Journal of Virology; 56; 779-789.

Osten P, Grinevich V, Cetin A. (2007). Viral Vectors: A Wide Range of Choices and High Level Services. HEP; 178; 177-202.

Pages JC, Danos O. (2003). In Rolland A, Sullivan SM. Retrovectors Go Forward. Pharmaceutical Gene Delivery Systems. Eastern Hemisphere Distribution, USA. Chapter 9.

Pillai O, Panchagnula R. (2001). Polymers in drug delivery. Current Opinion in Chemical Biology; 5: 447-451.

Pitt WG, Husseini GA, Staples BJ. (2004). Ultrasonic Drug Delivery - A General Review. Expert Opinion on Drug Delivery; $1 ; 37-56$.

Prokop A, Davidson JM. (2007). In Lanza R, Langer R, Vacanti J (ed). Gene Delivery into Cells and Tissues. Princeples of Tissue Engineering. Elsevier Academic Press, ABD; pp: 493-515.

Restifo NP, Dudley ME, Rosenberg SA. (2012). Adoptive immunotherapy for cancer: harnessing the T cell response. Nature Reviews Immunology; 12; 269-281. 
Reynolds PN, Feng M, Curiel DT. (1999). Chimeric viral vectors - the best of both worlds? Molecular Medicine Today; 4; 25-31.

Robbins PD, Ghivizzani SC. (1998). Viral Vectors for Gene Therapy. Pharmacology $\mathcal{E}$ Therapeutics; 80 ; 35-47.

Schatzlein AG. (2003). Targeting of Synthetic Gene Delivery Systems. Journal of Biomedicine and Biotechnology: 2; 149-158.

Scheier B, Amaria R, Lewis K, Gonzalez R. (2011). Novel therapies in melanoma. Immunotherapy; 3;1461-1469.

Somiari S, Glasspool-Malone J, Drabick JJ, Gilbert RA, Heller R, Jaroszeski MJ, Malone RW. (2000). Theory and in vivo Application of Electroporative Gene Delivery. Molecular Therapy: $2 ; 178-187$.

Suda T, Liu D. (2007). Hydrodynamic Gene Delivery: Its Principles and Applications. Molecular Therapy; 15: 2063-2069.

Sullivan SM. (2003). In Sullivan SM, Rolland A (ed). Introduction to Gene Therapy and Guidelines to Pharmaceutical Development. Pharmaceutical Gene Delivery Systems. Eastern Hemisphere Distribution, USA; pp. 17-31.

Tunçay M, Çalış S. (1999) İlaç taşıyıcı sistemlerde kullanılan biyoparçalanabilir sentetik ve doğal polimerler. FABAD J. Pharm. Sci; 24: 109-123.

Uyechi-O’Brien LS, Szoka FC (2003). In Rolland A, Sullivan SM (ed). Mechanisms for Cationic Lipids in Gene Transfer. Pharmaceutical Gene Delivery Systems. Eastern Hemisphere Distribution, USA. Chapter 4.

Wang X, Cao L, Wang Y, Wang X, Liu N, You Y. (2012). Regulation of let-7 and its target oncogenes (Review). Oncology Letters; 3; 955-960.

Ward BB, Baker Jr. JR. (2008). In Majoros IJ, Baker Jr. JR (ed). Targeted Drug Delivery in General, New Technology in Medicine. Dendrimer Based Nanomedicine. Pan Stanford Publishing, USA. pp: 1-16.

Willemejane J, Mir LM. (2009). Physical Methods of Nucleic Acid Transfer: General Concepts and Applications. British Journal of Pharmacology; 157; 207-219.

Witlox MA, Lamfers ML, Wuisman PI, Curiel DT, Siegal GP. (2007). Evolving gene therapy approaches for osteosarcoma using viral vectors: review. Bone; 40; 797-812.

Wong SY, Pelet JM, Putnam D. (2007). Polymer systems for gene delivery-Past, present, and future. Progress in Polymer Science; 32; 799-837.

Wunderbaldinger P, Bogdanov Jr A, Weissleder R. (2000). New approaches for imaging in gene therapy. European Journal of Radiology; 34; 156-165.

Yaron Y, Kramer RL, Johnson MP, Evans MI. (1997). Gene therapy: Is the Future Here Yet? Fetal Diagnosis and Therapy; 24; 179-197.

Yi Y, Noh MJ, Lee KH. (2011). Current Advances in Retroviral Gene Therapy. Current Gene Therapy: $11 ; 218-228$.

Zhang X, Edwards JP, Mosser DM. (2009). The Expression of Exogenous Genes in Macrophages: Obstacles and Opportunities. Methods in Molecular Biology; 531; 1-16. 
470 Recent Advances in Novel Drug Carrier Systems

Ziello JE, Huang Y, Jovin IS. (2010). Celular Endocytosis and Gene Delivery. Molecular Medicine; 16; 222-229. 